\title{
Asthma in childhood
}

\author{
Fernando Maria de Benedictis ${ }^{1}$ and Marina Attanasi ${ }^{2}$ \\ Affiliations: ${ }^{1}$ Dept of Pediatrics, Salesi Children's Hospital, Ancona, Italy. ${ }^{2}$ Dept of Paediatrics, University of \\ Chieti, Chieti, Italy.
}

Correspondence: Fernando Maria de Benedictis, Salesi Children's Hospital, 11, via Corridoni, 60123 Ancona, Italy. E-mail: pediatriaAfmdebenedictis.it

ABSTRACT Several topics on childhood asthma were addressed in the Paediatric Clinical Year in Review session at the 2015 European Respiratory Society International Congress. With regard to the relationship between lower respiratory tract infections and asthma, it emerges that is the number of respiratory episodes in the first years of life, but not the particular viral trigger, to be associated with later asthma development. Understanding which characteristics of individual patients are associated with an increased risk for asthma exacerbation is a critical step to implement strategies preventing these seasonal events. Recent data suggest the possibility that exhaled volatile organic compounds may qualify as biomarkers in detecting early signs of asthma. Adding information of exhaled volatile organic compounds and expression of inflammation genes to a clinical tool significantly improves asthma prediction in preschool wheezy children. Personal communication with children and adolescents is likely more important than the tools actually used for monitoring asthma. Systemic corticosteroids do not affect the long-term prognosis in children with first viral-induced wheezing episode and should be used cautiously during acute episodes. Finally, stress and a polymorphism upstream of a specific gene are both associated with reduced bronchodilator response in children with asthma.

0 @ERSpublications

New research reports must be integrated into medical communication to assist clinicians to improve asthma management http://ow.ly/XACHS

\section{Introduction}

During the 2015 European Respiratory Society International Congress in Amsterdam, the Netherlands, the main topics on childhood asthma were addressed in the Paediatric Clinical Year in Review session. This review summarises new information on asthma and wheezing arising from recently published peer-reviewed articles that have been presented at that meeting.

\section{Respiratory tract infections in early life and later asthma}

The observation that lower respiratory tract infections in the first years of life are associated with recurrent wheezing and later asthma $[1,2]$ led to focus on the role and pathogenetic mechanisms of viral agents such as rhinoviruses and respiratory syncytial virus in asthma development [3,4]. The role of bacterial agents in eliciting asthmatic symptoms in young children and in increasing the risk of asthma development has been emphasised as well. Indeed, the normal microbiota exerts profound effects on the mucosal immune system and may be determinant in human physiology and disease [5]. In the Copenhagen Prospective Study on Asthma in Childhood (COPSAC), neonates colonised in the hypopharyngeal region with Streptococcus pneumoniae, Haemophilus influenzae, or Moraxella catarrhalis, or with a combination of these organisms, were at increased risk for recurrent wheeze and asthma early in life [6]. In the same cohort, the authors found a significant association between bacterial infection of the airways and acute wheezy episodes, which was independent of viral infections [7].

Received: Nov 022015 | Accepted after revision: Dec 152015

Conflict of interest: None declared.

Provenance: Submitted article, peer reviewed.

Copyright OERS 2016. ERR articles are open access and distributed under the terms of the Creative Commons Attribution Non-Commercial Licence 4.0. 
Altered epithelial immune response to rhinoviruses infection in asthmatics is likely to play a critical role in relation to respiratory tract infections. EDWARDS et al. [8] showed that bronchial epithelial cells from atopic asthmatic children had impaired interferon- $\beta$ and $-\gamma$ levels in response to rhinoviruses infection. A deficient production of interferon by bronchial epithelial cells in response to rhinoviruses was found not only in atopic and non-atopic asthmatics, but also in atopic controls [9], thus suggesting that the impaired immune response of the epithelium after viral infection was more likely related to the T-helper cell type 2 inflammatory pattern in the airways than to intrinsic characteristic of asthma.

In order to explore if pre-existing susceptibility and individual inflammatory response to respiratory tract infections may be important risk factors for asthma rather than the specific trigger agent, BøNNELYKKE et al. [10] evaluated 313 children from the COPSAC high-risk birth cohort. Respiratory symptoms were recorded in a daily diary until age 3 years; a visit was scheduled after each 3-day episode of troublesome lung symptoms; airway secretion samples to detect nine viruses and three bacteria were obtained in that occasion; lung function was measured at 1 month of age; skin prick test and specific IgE levels against the main allergens were obtained at 6 years of age; finally, asthma at age 7 years was diagnosed according with international guidelines. The authors found that the number of respiratory episodes in the first years of life, but not the specific viral trigger, was associated with asthma at 7 years (figure 1). This was the first demonstration that the microbial trigger is not an important risk factor for later asthma development. These data, together with the recent report of interaction between early respiratory tract infections and the asthma locus at chromosome 17q21 [11], address future research towards studies on individual susceptibility of lower respiratory tract infections.

\section{Systemic steroids for treatment of acute wheezing}

Overall, randomised controlled trials (RCTs) on the efficacy of systemic corticosteroids in the treatment of early wheezing had not reported clinical efficacy $[12,13]$. Unlike these studies, a post hoc analysis of RCT data reported that oral prednisolone during the first rhinoviruses-induced wheezing episode reduced the risk of recurrent wheezing over the next 7 years [14]. In order to confirm these data, JARTTI et al. [15] performed a prospective RCT in young children with moderate-to-severe wheeze episodes caused by rhinoviruses. After confirming rhinoviruses infection from nasopharyngeal aspirate by PCR technique, 79 children aged 323 months with a first wheezing episode were randomised to receive oral prednisolone (first dose of $2 \mathrm{mg} \cdot \mathrm{kg}^{-1}$, followed by $2 \mathrm{mg} \cdot \mathrm{kg}^{-1}$ per day in two divided doses for 3 days) or placebo, and then were followed for 12 months. The three primary outcomes were the occurrence of a new physician-confirmed wheezing episode during the 2-month follow-up, the number of physician confirmed wheezing episodes during the 12month follow-up, and the initiation of regular controller medication for asthma symptoms during the 12month follow-up. Prednisolone treatment had mild benefits on some secondary short-term outcomes (less cough, rhinitis), but did neither reduce hospitalisation nor prevent long-term wheezing episodes (figure 2). The results of subgroup analyses showed no selective treatment effect in infants with atopy but, in children, with high viral loads ( $>7000$ copies $\cdot \mathrm{mL}^{-1}$ ) occurrence of new wheezing episodes during the follow-up period was lower in the active group than in the placebo group. Despite some mild benefits in short-term outcomes, prednisolone treatment cannot therefore be routinely recommended for children with their first acute virus-induced wheezing episode. Systemic steroids should be considered only in young children admitted to a hospital with features strongly suggestive of atopic asthma (e.g. a combination of multi-trigger wheeze, severe

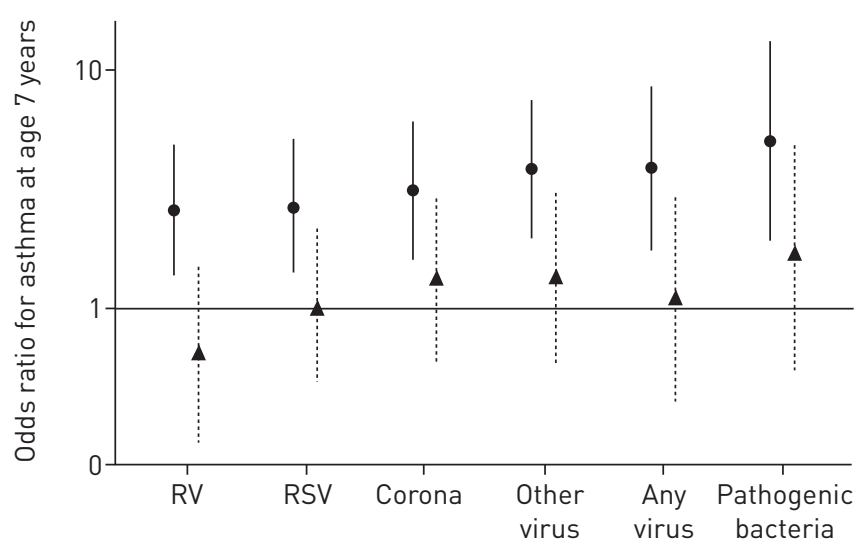

FIGURE 1 Risk of asthma at age 7 years in relation to viral or bacterial respiratory tract infection in the first 3 years of life. Data are presented as crude (circles) and adjusted (triangles; adjusted for the total number of respiratory episodes from which an aspirate was taken) odd ratios with $95 \%$ confidence intervals. RV: rhinovirus; RSV: respiratory syncytial virus. Reproduced from [10] with permission from the publisher. 


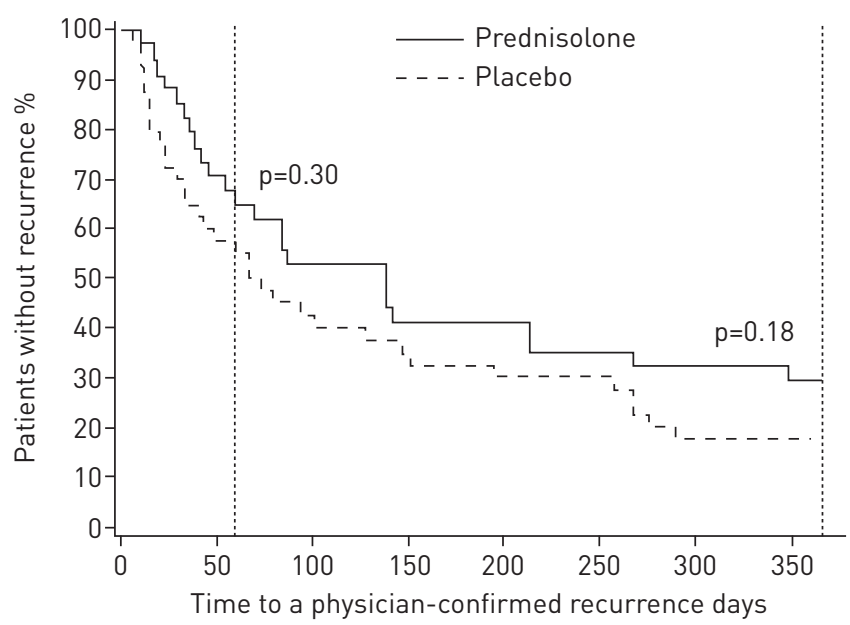

FIGURE 2 Time to a new physician-confirmed wheezing episode in children receiving prednisolone or placebo. The 2-month time-point, which was one of the primary outcomes, and the 12-month time-point are marked with vertical lines. Reproduced from [15] with permission from the publisher.

eczema and a family history of atopic asthma) or with very severe bronchodilator-unresponsive wheeze who appear to need high dependency or intensive care [16].

\section{Viral wheeze, exhaled biomarker profiles and prediction of asthma}

The analysis of exhaled volatile organic compounds (VOC) is a noninvasive 'omics approach of airway inflammation. In adults, VOC profile is associated with inflammatory activity and can differentiate patients with asthma from healthy controls [17]. Furthermore, children with preschool wheeze have different VOC profile when compared with healthy children [18], as a possible consequence of a prolonged inflammatory response associated with viral infection. In order to challenge if acute preschool wheeze is accompanied by a differential VOC profile and if this profile is maintained after resolution of symptoms in children with rhinoviruses infection, VAN DER SCHEE et al. [19] assessed exhaled VOC in 81 wheezy children during and after recovery of an acute episode of the lower respiratory tract and in 97 controls. Wheezy children showed a significantly different VOC profile compared with controls, regardless of the presence or absence of rhinoviruses infection. After recovery, discriminative accuracy was maintained only in children with rhinoviruses-induced wheeze, whereas it dropped significantly in those with non-rhinoviruses-induced wheeze. These findings clearly indicate that exhaled VOC may capture a prolonged inflammatory response associated with symptomatic rhinoviruses infection. As rhinoviruses-induced wheeze is associated with a 10 -fold increased risk of future diagnosis of asthma [3, 4], exhaled VOC may be candidate biomarkers to noninvasively detect early signs of asthma in preschool children.

A reliable asthma prediction is difficult in wheezy preschool children. Multiple predictive tools have been developed to identify young children at high risk for asthma development. Among them, the Asthma Predictive Index (API) is increasingly used in clinical practice, however it has a relatively poor positive predictive value [20]. In recent years, noninvasive tools have been developed to analyse inflammatory markers both in exhaled breath, as exhaled nitric oxide fraction (FeNO) [21] and VOC [22], and in the exhaled breath condensate (EBC) [23]. Moreover, analysis of expression of genes associated with inflammation and measures of airway resistance resulted in having an additional diagnostic role in identifying wheezing phenotypes [24, 25]. In previous studies, these markers have been separately associated with childhood asthma $[26,27]$. In order to assess whether exhaled biomarkers, expression of inflammatory genes and early lung function measurements can improve a reliable asthma prediction in preschool wheezy children, KLAASSEN et al. [28] evaluated 202 children, aged 2-4 years, from the Asthma Detection and Monitoring (ADEM) cohort, and prospectively followed them yearly until 6 years of age. At the time of recruitment, clinical patient characteristics, airway resistance, EBC, VOC and RNA from peripheral blood mononuclear cells were obtained. In the discriminative analysis, data on EBC and airway resistance did not improve the area under the curve (AUC) when added to the API. However, the addition 


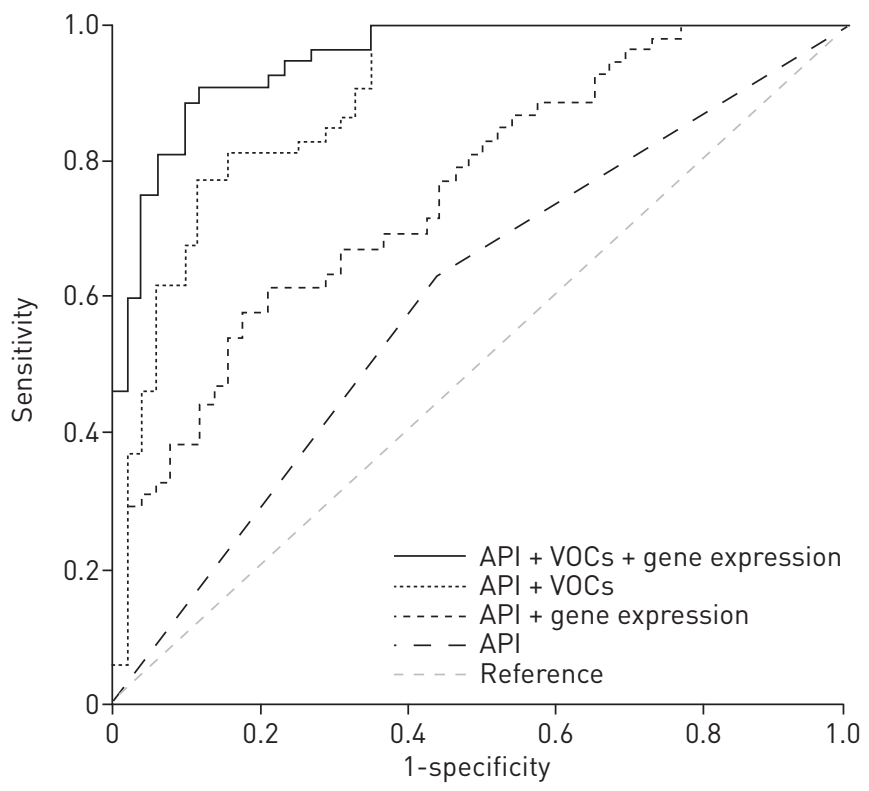

FIGURE 3 Receive operating characteristic curves for volatile organic compounds (VOC), expression of inflammatory genes, and the Asthma Predictive Index (API) in a combined model in the discovery set. Reproduced from [28] with permission from the publisher.

of exhaled VOC and gene expression (toll-like receptor 4, catalase and tumour necrosis factor- $\alpha$ ) to the API status improved the asthma prediction by $28 \%$ and $17 \%$, respectively, compared with the clinical model (figure 3). The combined model (API plus VOC plus gene expression) accurately classified $89 \%$ of children, while the API alone correctly classified only $60 \%$ of them. This innovative, combined approach sheds new light on the field of asthma prediction and proposes as a promising diagnostic tool for asthma. Further studies are however necessary to evaluate the clinical relevance of these data.

\section{Asthmatic exacerbations}

Asthma exacerbations are a major factor responsible for the morbidity and even mortality of children with asthma [29]. Given the importance of exacerbations to the overall burden of asthma, attention has focused on the causes of these events, including viral respiratory tract infections, environmental allergens, pollutants and stress [30]. Although asthma exacerbations can occur at any time during the year, seasonal patterns exist in children [31], with an increased risk in the autumn [32]. Therefore, understanding which characteristics are associated with an increased risk for asthma exacerbation is an important step to prevent these events and might include seasonal changes of treatment. In order to clarify this aspect, TEACH et al. [33] conducted a retrospective analysis of 400 control group participants, aged 6-20 years, from two recent studies, the Asthma Control Evaluation trial [34] and the Inner City Anti-IgE Therapy for Asthma trial [35]. Univariate and multivariate pooled analyses were applied to determine whether demographic and historical factors, allergic sensitisation measures, FeNO, spirometric values, asthma control measures and asthma treatment requirements were associated with seasonal exacerbations. Of the participants, $37.5 \%$ experienced a defined asthma exacerbation, with the highest frequency of $28.8 \%$ in the autumn, $19.9 \%$ in the spring, $15.9 \%$ in the winter, and $14.5 \%$ in the summer. For the full-year univariate analysis, factors most associated with exacerbations included an exacerbation in the prior year, decreased pulmonary function measures, higher requirements for inhaled corticosteroids (ICS) and allergy. The strongest predictors for autumn exacerbations included an exacerbations during the prior season, markers of allergy such as allergen-specific IgE levels to house dust mite and cockroach, increased FeNO levels and decreased lung function. Only two factors predicted winter exacerbations: decreased pulmonary function values and an exacerbation during the previous season. Spring exacerbations were most closely associated with an exacerbation during the previous year, but not in the previous season. Finally, summer exacerbations were related to impaired pulmonary function, sensitisation to Alternaria spp., increased blood eosinophil counts, higher ICS doses, and an exacerbation in the previous season. Multivariate analysis showed that the best predictive factors were a history of an exacerbation in the last season for autumn and winter exacerbations, while the asthma treatment requirements were critical for spring and summer exacerbations. Although the results of this study require further validation by studies involving other populations of children and adolescents with asthma and a longer follow-up, information on individual patients might be beneficial in strategies to prevent seasonal events and might help to achieve a more complete, systematic and personalised approach to asthma management. 
Two prior Inner-City Asthma Consortium (ICAC) studies found that the frequency of exacerbations was reduced with higher daily doses of ICS [34] or with omalizumab [35] when added to year-round guidelines-based treatment. Because continuous treatment with both therapeutic modalities could conduct to certain risks and increased costs, innovative strategies of therapy were considered. In the Preventative Omalizumab or Step-up Therapy for Fall Exacerbations (PROSE) study, TEACH et al. [36] determined whether a targeted strategy of beginning therapy with omalizumab 4-6 weeks before the start of school and continuing it for the next 4 months could be more efficacious than placebo or an ICS boost in preventing autumn asthma exacerbations among children already receiving guidelines-based therapy. The study showed that inner-city children might benefit from the addition of omalizumab to ongoing guidelines-based therapy before autumn to prevent exacerbations if they have severe disease and particularly if they have a history of a recent exacerbation, irrespective of disease severity.

\section{Monitoring asthma}

The importance of monitoring asthma and adjusting treatment according to control is a fundamental part of asthma management. Numerous tools can be used, including spirometry, biomarkers, airway hyperresponsiveness and validated questionnaires. However, the evidence of the utility of most of these tools is lacking, particularly in children, and there is limited evidence as to how the various components of control should be integrated [37]; in addition, studies tend to evaluate each measure in isolation. In order to evaluate possible benefits of web-based monitoring, Voorend-VAn BERgen et al. [38] conducted a multicentre, prospective, partly blinded, parallel group, three-arm RCT on monitoring strategies in 268 asthmatic children aged 4-18 years, with a follow-up of 1 year (Better Asthma Treatment: Monitoring with ACT and Nitric oxide (BATMAN) study). Standard care was based on the Asthma Control Test (ACT) [39] or Childhood Asthma Control Test (C-ACT) for younger children [40], evaluated every 4 months at routine clinic visits; the two alternative strategies were a web-based ACT/C-ACT score measure with treatment adapted monthly, and the addition of FeNO measurement to the 4-month visits. After 1 year follow-up, neither monthly web-based monitoring with the ACT/C-ACT nor regular measurement of FeNO increased symptom-free days (main outcome) in asthmatic children. However, there was a clinically relevant decrease of ICS dose in the monthly ACT/C-ACT group, and a modest increase of the asthma control score in children younger than 12 years in the FeNO group; no other measures of asthma control improved as result of FeNO monitoring. The higher frequency of healthcare contacts obtained by administering the ACT/C-ACT monthly might partially explain the success of this approach in reducing the ICS dose. Finding ways to effectively communicate with young people and use advances in technology to make healthcare interactions meaningful is likely an important issue that was properly emphasised in the accompanying editorial [41].

\section{Stress and asthma}

Stress has been associated with reduced expression of the gene for the $\beta_{2}$-adrenergic receptor (ADRB2) in leukocytes from children with asthma [42]. The finding that acute stress was not associated with ADRB2 expression in the absence of chronic stress suggests that the down-regulation of the ADRB2 is due to persistent secretion of the hormonal products of the hypothalamic-pituitary-adrenocortical axis, including catecholamines, in chronically stressed children. It is also known that children from Puerto Rico have lower bronchodilator response and greater asthma morbidity than children from other ethnic groups, an observation that may be explained by either the presence of allelic variants in ADRB2 [43] or increased psychosocial stress in this population [44]. The gene encoding the pituitary adenylate cyclase-activating polypeptide 1 (PACAP 1) receptor type 1 (ADCYAP1R1) has been previously implicated in anxiety [45] and asthma [46], but it is actually unknown whether dysfunction of the PACAP 1 pathway explains the potential effect of stress on the ADRB2. In order to evaluate whether stress or ADCYAP1R1 variation was associated with reduced bronchodilator response in children with asthma, BREHM et al. [47] performed a cross-sectional study in 351 asthmatic children living in Puerto Rico and in a replication study cohort (RIPRAC). Bronchodilator response was evaluated according with standard criteria. Children's stress resulting from violence during the preceding 6 months and maternal stress were estimated by specific questionnaires. In addition, polymorphisms in ADCYAP1R1 were genotyped. High levels of personal and household stress were associated with lower bronchodilator response in children with asthma. Furthermore, a single-nucleotide polymorphism in ADCYAP1R1 (rs34548976) was associated with reduced bronchodilator response. This polymorphism is also associated with reduced expression of the gene for $A D R B 2$ in $\mathrm{CD}^{+}$lymphocytes of subjects with asthma, and it affects brain connectivity of the amygdala and insula, that is a biomarker of anxiety. The results of the study are consistent with a negative effect of single-nucleotide polymorphism rs34548976 on bronchodilator response through neuro-hormonal mechanisms leading to a down-regulation of $A D R B 2$ in highly stressed children. These scientific findings are also of major public health significance, as they underscore the importance of policies that support a healthy family and community environment [48]. 


\section{Summary}

A series of hot topics on childhood asthma was presented. With regard to these topics, the future directions on asthma research should focus on the mechanisms of the susceptibility and exaggerated response to lower respiratory tract infections. Because bacteria may contribute to the burden of wheezy symptoms, trials with antibiotic therapy will be required to determine potential clinical effects. Future research should also focus on the implication for clinical practice and costs when noninvasive biomarkers are considered for asthma prediction. Furthermore, information on characteristics of individual patients might be beneficial in strategies to prevent seasonal exacerbations, and should be evaluated in studies addressed to achieve a personalised approach to asthma. Finally, new research reports need to be integrated into medical communication to assist clinicians in order to improve asthma management and reduce morbidity and mortality.

\section{References}

1 Stein RT, Sherrill D, Morgan WJ, et al. Respiratory syncytial virus in early life and risk of wheeze and allergy by age 13 years. Lancet 1999; 354: 541-545.

2 Sigurs N, Bjarnason R, Sigurbergsson F, et al. Respiratory syncytial virus bronchiolitis in infancy is an important risk factor for asthma and allergy at age 7. Am J Respir Crit Care Med 2000; 161: 1501-1507.

3 Lemanske RF Jr, Jackson DJ, Gangnon RE, et al. Rhinovirus illnesses during infancy predict subsequent childhood wheezing. J Allergy Clin Immunol 2005; 116: 571-577.

4 Jackson DJ, Gangnon RE, Evans MD, et al. Wheezing rhinovirus illnesses in early life predict asthma development in high-risk children. Am J Respir Crit Care Med 2008; 178: 667-672.

5 Castro-Nallar E, Shen Y, Freishtat RJ, et al. Integrating microbial and host transcriptomics to characterize asthma-associated microbial communities. BMC Med Genomics 2015; 8: 50.

6 Bisgaard $\mathrm{H}$, Hermansen $\mathrm{MN}$, Buchvald $\mathrm{F}$, et al. Childhood asthma after bacterial colonization of the airway in neonates. N Engl J Med 2007; 357: 1487-1495.

7 Bisgaard H, Hermansen MN, Bønnelykke K, et al. Association of bacteria and viruses with wheezy episodes in young children: prospective birth cohort study. BMJ 2010; 341: c4978.

8 Edwards MR, Regamey N, Vareille M, et al. Impaired innate interferon induction in severe therapy resistant atopic asthmatic children. Mucosal Immunol 2013; 6: 797-806.

9 Baraldo S, Contoli M, Bazzan E, et al. Deficient antiviral immune responses in childhood: distinct roles of atopy and asthma. J Allergy Clin Immunol 2012; 130: 1307-1314.

10 Bønnelykke K, Vissing NH, Sevelsted A, et al. Association between respiratory infections in early life and later asthma is independent of virus type. J Allergy Clin Immunol 2015; 136: 81-86.

11 Caliskan M, Bochkov YA, Kreiner-Møller E, et al. Rhinovirus wheezing illness and genetic risk of childhood-onset asthma. N Engl J Med 2013; 368: 1398-1407.

12 Oommen A, Lambert PC, Grigg J. Efficacy of a short course of parent-initiated oral prednisolone for viral wheeze in children aged 1-5 years: randomised controlled trial. Lancet 2003; 362: 1433-1438.

13 Panickar J, Lakhanpaul M, Lambert PC, et al. Oral prednisolone for preschool children with acute virus-induced wheezing. N Engl J Med 2009; 360: 329-338.

14 Jartti T, Lehtinen P, Vanto T, et al. Evaluation of the efficacy of prednisolone in early wheezing induced by rhinovirus or respiratory syncytial virus. Pediatr Infect Dis J 2006; 25: 482-488.

15 Jartti T, Nieminen R, Vuorinen T, et al. Short- and long-term efficacy of prednisolone for first acute rhinovirus-induced wheezing episode. J Allergy Clin Immunol 2015; 135: 691-698.e9.

16 de Benedictis FM, Bush A. Corticosteroids in respiratory diseases in children. Am J Respir Crit Care Med 2012; 85: 12-23.

17 Dragonieri S, Schot R, Mertens BJ, et al. An electronic nose in the discrimination of patients with asthma and controls. J Allergy Clin Immunol 2007; 120: 857-862.

18 van de Kant KDG, van Berkel JJ, Jöbsis Q, et al. Exhaled breath profiling in diagnosing wheezy preschool children. Eur Respir J 2013; 41: 183-188.

19 van der Schee MP, Hashimoto S, Schuurman AC, et al. Altered exhaled biomarker profiles in children during and after rhinovirus-induced wheeze. Eur Respir J 2015; 45: 440-448.

20 Castro-Rodriguez JA. The asthma predictive index: a very useful tool for predicting asthma in young children. J Allergy Clin Immunol 2010; 126: 212-216.

21 Pijnenburg MWH, De Jongste JC. Exhaled nitric oxide in childhood asthma: a review. J Allergy Clin Immunol 2008; 38: 246-259.

22 Van de Kant KD, van der Sande LJ, Jobsis Q, et al. Clinical use of exhaled volatile organic compounds in pulmonary diseases: a systematic review. Respir Res 2012; 13: 117.

23 Kazani S, Israel E. Exhaled breath condensate in asthma: diagnostic and therapeutic implications. J Breath Res 2010; 4: 047001.

24 Robroeks CM, Rijkers GT, Jobsis Q, et al. Increased cytokines, chemokines and soluble adhesion molecules in exhaled breath condensate of asthmatic children. Clin Exp Allergy 2010; 40: 77-84.

25 Kooi EM, Schokker S, van der Molen T, et al. Airway resistance measurements in pre-school children with asthmatic symptoms: the interrupter technique. Respir Med 2006; 100: 955-964.

26 Dallinga JW, Robroeks CM, van Berkel JJ, et al. Volatile organic compounds in exhaled breath as a diagnostic tool for asthma in children. Clin Exp Allergy 2010; 40: 68-76.

27 van de Kant KD, Klaassen EM, Jöbsis Q, et al. Wheezing in preschool children is associated with increased levels of cytokines/chemokines in exhaled breath condensate. J Allergy Clin Immunol 2010; 126: 669-671.

28 Klaassen EM, van de Kant KD, Jöbsis Q, et al. Exhaled biomarkers and gene expression at preschool age improve asthma prediction at 6 years of age. Am J Respir Crit Care Med 2015; 191: 201-207.

29 Moorman JE, Akinbami LJ, Bailey CM, et al. National surveillance of asthma: United States, 2001-2010. Vital Health Stat 2012; 35: 1-67.

30 Sears MR. Epidemiology of asthma exacerbations. J Allergy Clin Immunol 2008; 122: 662-670. 
31 Cohen HA, Blau H, Hoshen M, et al. Seasonality of asthma: a retrospective population study. Pediatrics 2014; 133: e923-e932.

32 Sears MR, Johnston NW. Understanding the September asthma epidemic. J Allergy Clin Immunol 2007; 120: 526-529.

33 Teach SJ, Gergen PJ, Szefler SJ, et al. Seasonal risk factors for asthma exacerbations among inner city children. J Allergy Clin Immunol 2015; 135: 1465-1473.

34 Szefler SJ, Mitchell H, Sorkness CA, et al. Management of asthma based on exhaled nitric oxide in addition to guideline based treatment for inner-city adolescents and young adults: a randomized controlled trial. Lancet 2008; 372: 1065-1072.

35 Busse WW, Morgan WJ, Gergen PJ, et al. Randomized trial of omalizumab (anti-IgE) for asthma in inner-city children. N Engl J Med 2011; 364: 1005-1015.

36 Teach SJ, Gill MA, Togias A, et al. Preseasonal treatment with either omalizumab or an inhaled corticosteroid boost to prevent fall asthma exacerbations. J Allergy Clin Immunol 2015; 136: 1476-1485.

37 Pijnenburg MW, Baraldi E, Brand PL, et al. Monitoring asthma in children. Eur Respir J 2015; 45: 906-925.

38 Voorend-van Bergen S, Vaessen-Verberne AA, Brackel HJ, et al. Monitoring strategies in children with asthma: a randomised controlled trial. Thorax 2015; 70: 543-550.

39 Nathan RA, Sorkness CA, Kosinski M, et al. Development of the asthma control test: a survey for assessing asthma control. J Allergy Clin Immunol 2004; 113: 59-65.

40 Liu AH, Zeiger R, Sorkness C, et al. Development and cross-sectional validation of the Childhood Asthma Control Test. J Allergy Clin Immunol 2007; 119: 817-825.

41 Fleming L. Monitoring asthma in children: what does BATMAN tell us? Thorax 2015; 70: 517-518.

42 Miller GE, Chen E. Life stress and diminished expression of genes encoding glucocorticoid receptor and beta2-adrenergic receptor in children with asthma. Proc Natl Acad Sci USA 2006; 103: 5496-5501.

43 Choudhry S, Ung N, Avila PC, et al. Pharmacogenetic differences in response to albuterol between Puerto Ricans and Mexicans with asthma. Am J Respir Crit Care Med 2005; 171: 563-570.

44 Cohen RT, Canino GJ, Bird HL, et al. Violence, abuse, and asthma in Puerto Rican children. Am J Respir Crit Care Med 2008; 178: 453-459.

45 Jovanovic T, Norrholm SD, Davis J, et al. PAC1 receptor (ADCYAP1R1) genotype is associated with dark-enhanced startle in children. Mol Psychiatry 2013; 18: 742-743.

46 Chen W, Boutaoui N, Brehm JM, et al. ADCYAP1R1 and asthma in children. Am J Respir Crit Care Med 2013; 187: 584-588.

47 Brehm JM, Ramratnam SK, Tse SM, et al. Stress and bronchodilator response in children with asthma. Am J Respir Crit Care Med 2015; 192: 47-56.

48 Lang JE. Stress, ADCYAP1R1, and childhood asthma. No sigh of relief. Am J Respir Crit Care Med 2015; 192: 3-5. 